\title{
Some Combinatorial Optimization Problems for Weak-Bisplit Graphs
}

\author{
Mihai Talmaciu, Elena Nechita \\ University of Bacău, Department of Mathematics and Informatics, \\ 8 Spiru Haret, Bacău, Romania, \\ mtalmaciu@ub.ro, enechita@ub.ro
}

\begin{abstract}
There exists linear algorithms to recognize weak-bisplit graphs and NP-complete optimization problems are efficiently resolved for this class of graphs. In this paper, using weak-decomposition, we give necessary a sufficient conditions for a graph to be weak-bisplit, bi-cograph, weak-bisplit cograph. We also give an algorithm $O(n+m)$ to determine, the density of a weak-bisplit cograph and we calculate directly the domination number for this class of graphs.
\end{abstract}

Keywords: Weak-bisplit graphs, Bi-cographs, Weak-bisplit cographs.

\section{Introduction}

Throughout this paper $G=(V, E)$ is a simple (i.e. finite, undirected, without loops and multiple edges) graph [2]. Let $c o-G=\bar{G}$ denote the complement graph of $G$. For $U \subseteq V$ let $G(U)$ denote the subgraph of $G$ induced by $U$. By $G-X$ we mean the graph $G(V-X)$, whenever $X \subseteq V$, but we often denote it simply by $G-v .(\forall v \in V)$ when there is no ambiguity. If $v \in V$ is a vertex in $G$, the neighborhood $N_{G}(v)$ denotes the vertices of $G$ $v$ that are adjacent to $v$. We write $N(v)$ when the graph $G$ appears clearly from the context. The neighborhood of the vertex $v$ in the complement of the graph $G$ is denoted by $\bar{N}(v)$. For any subset $S$ of vertices in the graph $G$ the neighborhood of $S$ is $N(S)=\cup_{v \in S} N(v)-S$ and $N[S]=S \cup N(S)$. A clique is a subset of $V$ with the property that all the vertices are pairwise adjacent. The clique number (density) of $G$, denoted by $\omega(G)$ is the cardinal of the maximum clique. A clique cover is a partition of the vertices set such that each part is a clique. $\theta(G)$ is the cardinal of a smallest possible clique cover of $G$; it is called the clique cover number of $G$. A stable (or independent) set is a subset of $\mathrm{V}$ with the property that all the vertices are pairwise non-adjacent. The stability number of $G$ is $\alpha(G)=\omega(\bar{G})$; the chromatic number of $G$ is $\chi(G)=\theta(\bar{G})$.

A dominating set for a graph $G=(V, E)$ is a subset $D$ of $V$ such that every vertex not in $D$ is joined to at least one member of $D$ by some edge. The domination number $\gamma(G)$ is the number of vertices in a smallest dominating set for $G$.

By $P_{n}, C_{n}, K_{n}$ we mean a chordless path on $n \geq 3$ vertices, the chordless cycle on $n \geq 3$ vertices, and the complete graph on $n \geq 1$ vertices. If $e=x y \in E$, we also denote $x \sim y$; we also denote $x \times y$ whenever $x, y$ are not adjacent in $G$. A set $A$ is totally adjacent (non adjacent) with a set $B$ of vertices $(A \cap B=\phi)$ if $a b$ is (is not) edge, for any $a$ vertex in $A$ and any $b$ vertex in $B$; we note denote $A \sim B$ $(A \nsim B)$. A graph $G$ is $F$-free if none of its induced subgraphs is in $F$.

The subset $A \subset V$ is called a cutset if $G-A$ is not connected. If, in addition, none of the proper subsets of $A$ is a cutest, then $A$ is called a minimal cutset .

The paper is organized as follows. In Section 2 we give preliminary results. In Section 3 we give a characterization of weak-bisplit graphs.

\section{Preliminary Results}

At first, we recall the notion of weak component.

Definition 1. [10],[11],[12] $A$ set $A \subset V(G)$ is called a weak set of the graph $G$ if $N_{G}(A) \neq V(G)-A$ and $G(A)$ are connected. If $A$ is a weak set, maximal with respect to set inclusion, then $G(A)$ is called a weak component. For simplicity, the weak component $G(A)$ will be denoted by $A$.

Definition 2. [10],[11],[12] Let $G=(V, E)$ be a connected and non-complete graph. If $A$ is a weak set, then the partition $\{A, N(A), V$ - 
$A \cup N(A)\}$ is called a weak decomposition of $G$ with respect to $A$.

The name of "weak component" is justified by the following result.

Theorem 1. [10],[11],[12] Every connected and non-complete graph $G=(V, E)$ admits a weak component $A$ such that

$$
G(V-A)=G(N(A)+G(\bar{N}(A)) .
$$

Theorem 2. [10],[11],[12] Let $G=(V, E)$ be a connected and non-complete graph and $A \subset V$. Then $A$ is a weak component of $G$ if and only if $G(A)$ is connected and $N(A) \sim \bar{N}(A)$.

The next result, based on Theorem 1, ensures the existence of a weak decomposition in a connected and non-complete graph.

Theorem 3. If $G=(V, E)$ is a connected and non-complete graph, then $V$ admits a weak decomposition $(A, B, C)$, such that $G(A)$ is a weak component and $G(V-A)=G(B)+G(C)$.

Theorem 2 provides an $O(n+m)$ algorithm for building a weak decomposition for a noncomplete and connected graph.

\section{Algorithm for the weak decomposition of a graph [10], [12]}

Input: A connected graph with at least two nonadjacent vertices, $\mathrm{G}=(\mathrm{V}, \mathrm{E})$.

Output: A partition $\mathrm{V}=(\mathrm{A}, \mathrm{N}, \mathrm{R})$ such that $\mathrm{G}(\mathrm{A})$ is connected, $\mathrm{N}=\mathrm{N}(\mathrm{A}), \mathrm{A} \sim R=\bar{N}(A)$.

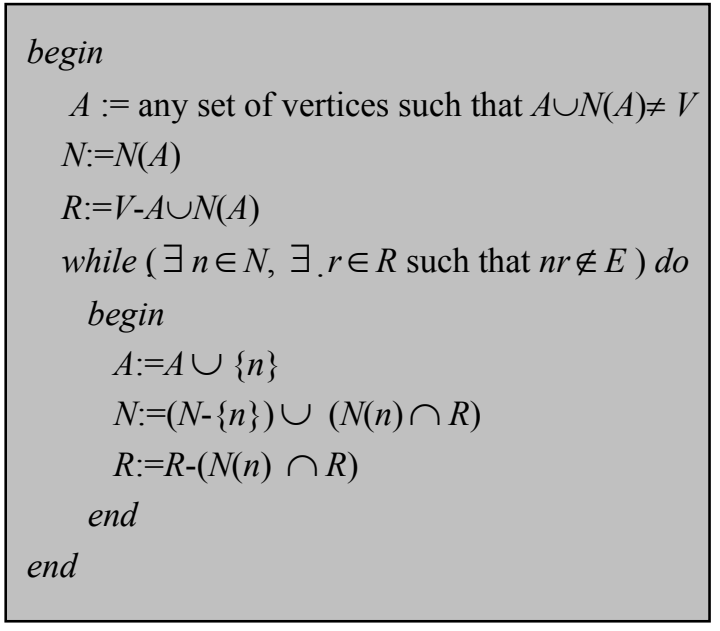

\section{The Results Concerning Weak- Bisplit Graphs}

\subsection{Basic properties}

\subsubsection{A new characterization of weak- bisplit graphs}

Definition 3. A graph $G$ is a weak bisplit graph if and only if it has an independent set $S$ such that every connected component of $G$ $S$ is a biclique (i. e. a complete bipartite subgraph).

A graph $\operatorname{Star}_{123}$ and the next graph $(\{a, b, c, d, e, f, g\},\{a b, b c, c d, c e, e f, f g\})$ are isomorphic.

In [3], Fouquet et al. introduced the notion of $K+S$-decomposition: a partition $\left(V_{1}, V_{2}\right)$ of the vertex set in a bipartite graph is a $K+S$ decomposition when the black vertices of $V_{1}$ are all adjacent to the white vertices of $V_{2}$ while there is no edge connecting a white vertex of $V_{1}$ to a black vertex of $V_{2}$. For a bipartite graph $G$, canonical decomposition [3] recursively applies $\mathrm{K}+\mathrm{S}$-decomposition.

The weak bisplit graphs are characterized (see [4]) by two excluded subgraphs $P_{7}$ and Star $_{123}$ as cographs are characterized by excluded $P_{4}$.

Weak bisplit graphs are designed in [3] to be completely decomposable with respect to canonical decomposition.

In [14] it is mentioned that the problem concerning the determination of the domination number (see [7]) and of the stability number (see [8]) is NP-complete, that the problem of the cliquewidth (see [6]) is unbounded, and that of recognition (see [1]) is also NP-complete.

In [5] it is given a linear algorithm to recognize weak-bisplit graphs is given and NP-complete optimization problems are efficiently resolved for this class of graphs.

A graph is weak bisplit [14] if and only if it is $\left\{P_{7}, C_{2 k+1}(k \geq 2)\right.$, Star $\left._{123}\right\}$-free.

Theorem 4. Let $G=(V, E)$ be a connected and incomplete graph and $(A, N, R)$ a weak decomposition with $G(A)$ the weak component. $G$ is weak-bisplit if and only if:

i) $G(R)$ is weak-bisplit; 
ii) $G(V-R)$ does not contain any of the graphs presented below:

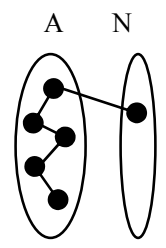

1)

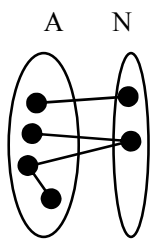

4)

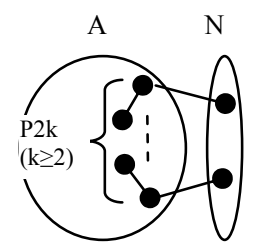

2)

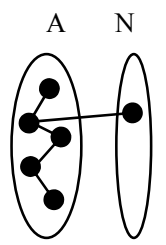

5)

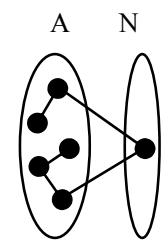

3)

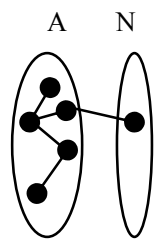

6)
Figure 1.

Proof. Let $(\mathrm{A}, \mathrm{N}, \mathrm{R})$ be a weak decomposition with $G(A)$ the weak component.

Let $\mathrm{G}$ be a weak-bisplit graph. We prove that $\mathrm{G}(\mathrm{V}-\mathrm{R})$ does not contain any of the graphs shown in Figure 1(1..6).

If G-R contains the graph in Figure1 (1), then G contains $P_{7}$, because $N \sim R$ and $A \nsim R$.

If G-R contains the graph in Figure 1 (2), then $\mathrm{G}$ contains $C_{2 k+1}(k \geq 2)$, because $N \sim R$ and $A \nsim R$.

If G-R contains the graph in Figure 1 (either 3 or 4 or 5 or 6 ), then $\mathrm{G}$ contains Star $_{123}$, because $N \sim R$ and $A \nsim R$.

Vice versa, we assume that G-R does not contain any of the graphs in Figure 1(1..6) and that $G(R)$ is weak-bisplit. We show that $\mathrm{G}$ is weak bisplit.

Suppose $\mathrm{G}$ is not weak-bisplit. Then $\mathrm{G}$ would contain $P_{7}$ or $C_{2 k+1}(k \geq 2)$ or $\operatorname{Star}_{123}$ as an induced subgraph.

If $\mathrm{G}$ contained $P_{7}$, then $P_{7} \subseteq G(R)$ or $V\left(P_{7}\right) \cap A \neq \Phi \quad$ and $\quad V\left(P_{7}\right) \cap N \neq \Phi$ and $V\left(P_{7}\right) \cap R \neq \Phi$.

Because $\quad N, R \neq \Phi \quad$ and $N \sim R$, $\left|V\left(P_{7}\right) \cap R\right|=1$ and $\left|V\left(P_{7}\right) \cap N\right| \leq 2$. If $\left|V\left(P_{7}\right) \cap N\right|=1$, then G-R contains the graph in Figure 1 (1). If $\left|V\left(P_{7}\right) \cap N\right|=2$, it contradicts the fact that $N=N_{G}(A)$ because there would be a vertex in $N$ that has no neighbor in $A$. If $\mathrm{G}$ contained $C_{2 k+1}(k \geq 2)$, then $C_{2 k+1} \subseteq G(R)$ or $V\left(C_{2 k+1}\right) \cap A \neq \Phi$ and $V\left(C_{2 k+1}\right) \cap N \neq \Phi$ and $V\left(C_{2 k+1}\right) \cap R \neq \Phi$. Because $N, R \neq \Phi$ and $\quad N \sim R, \quad\left|V\left(C_{2 k+1}\right) \cap R\right|=1 \quad$ and $\left|V\left(C_{2 k+1}\right) \cap N\right|=2$.

Since $N \sim R$, G-R contains the graph in Figure 1(2). If $\mathrm{G}$ contained $\operatorname{Star}_{123}$, then Star $_{123} \subseteq G(R)$ or $V\left(\right.$ Star $\left._{123}\right) \cap A \neq \Phi$ and $V\left(\operatorname{Star}_{123}\right) \cap N \neq \Phi \quad$ and $V\left(\operatorname{Star}_{123}\right) \cap R \neq \Phi$. Because $N, R \neq \Phi$ and $\quad N \sim R, \quad\left|V\left(\operatorname{Star}_{123}\right) \cap R\right|=1 \quad$ and $\left|V\left(\operatorname{Star}_{123}\right) \cap N\right| \leq 3$.

Let $\operatorname{Star}_{123}$ be the graph with $V\left(\operatorname{Star}_{123}\right)=\{a, b, c, d . e, f, g\} \quad$ and $E\left(\operatorname{Star}_{123}\right)=\{a b, b c, c d, c e, e f, f g\} . \quad$ If $a \in R$, then G-R contains the graph in Figure 1(5). If $b \in R$, then $a \in N$ and $a$ has no neighbor in A, which is impossible because $N=N_{G}(A)$. If $c \in R$, then $d \in N$ and $d$ has no neighbor in $\mathrm{A}$, which is impossible because $N=N_{G}(A)$. If $d \in R$, then G-R contains the graph in Figure 1 (3). If $e \in R$ then G-R contains the graph in Figure 1(4). If $f \in R$, then $g \in N$ and $g$ has no neighbor in $\mathrm{A}$, which is impossible because $N=N_{G}(A)$. If $g \in R$, then G-R contains the graph in Figure 1(6).

\subsubsection{A new characterization of bi-cographs}

A minimal super-class [13] of the class of bicographs is the class of weak-bisplit graphs.

A graph $\mathrm{Sun}_{4}$ and the next graph ( $\{a, b, c, d, e, f, g, h\},\{a e, b f, c g, d h, e f, f g, g h, h e\})$ are isomorphic.

Definition 4. [13] A graph is a bi-cograph if it is a bipartite graph that can be reduced to isolated vertices by recursively bicomplementing the edge set of all connected bipartite subgraphs, where the bicomplement of $(X, Y, E)$ is $(X, Y, X \times Y-E)$. 
A graph is bi-cograph [13] if and only if it is $\left\{P_{7}\right.$, Star $_{123}$, Sun $\left._{4}\right\}$-free.

Theorem 5. Let $G=(V, E)$ be a connected and incomplete graph and $(A, N, R)$ a weak decomposition with $G(A)$ the weak component. $G$ is bi-cograph if and only if:

i) $G-R$ does not contain any of the graphs in the Figure 2:

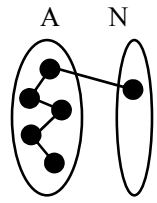

1)

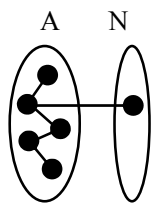

4)

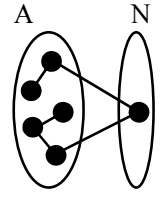

2)

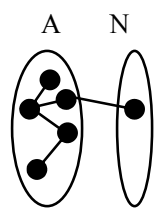

5)

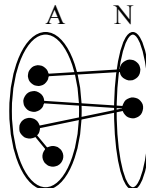

3)

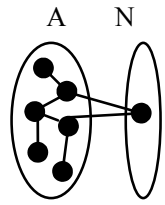

6)
Figure 2.

ii) $G(R)$ is bi-cograph.

Proof. Let $(\mathrm{A}, \mathrm{N}, \mathrm{R})$ be a weak decomposition with $G(A)$ the weak component. Let $G$ be a bi-cograph.

We show that G-R does not contain any of the graphs in Figure $2(1 \ldots 6)$. If G-R contains the graph in Figure 2 (1), then $G$ contains $P_{7}$, ecause $N \sim R$ and $A \nsim R$. If G$\mathrm{R}$ contains the graph in Figure 2 (either 2 or 3 or 4 or 5), then $\mathrm{G}$ contains Star $_{123}$, because $N \sim R$ and $A \nsim R$.

If G-R contains the graph in Figure 2 (6), then $\mathrm{G}$ contains $\mathrm{Sun}_{4}$, because $N \sim R$ and $A \nsim R$.

Vice versa, we assume that G-R does not contain any of the graphs in Figure 2(1..6) and that $G(R)$ is a bi-cograph and we show that $\mathrm{G}$ is a bi-cograph. We assume that $\mathrm{G}$ is not a bi-cograph. Then $\mathrm{G}$ should contain $P_{7}$ or $\operatorname{Star}_{123}$ or $\mathrm{Sun}_{4}$. If G contains $P_{7}$, then $P_{7} \subseteq G(R) \quad$ or $\quad V\left(P_{7}\right) \cap A \neq \Phi \quad$ and $V\left(P_{7}\right) \cap N \neq \Phi$ and $V\left(P_{7}\right) \cap R \neq \Phi$. Because $\quad N, R \neq \Phi \quad$ and $\quad N \sim R$, $\left|V\left(P_{7}\right) \cap R\right|=1$ and $\quad\left|V\left(P_{7}\right) \cap N\right| \leq 2$. If
$\left|V\left(P_{7}\right) \cap N\right|=1$, then G-R contains the graph in Figure 2 (1). If $\left|V\left(P_{7}\right) \cap N\right|=2$, then the fact that $N=N_{G}(A)$ is contradicted because there would be a vertex from $N$ that has no neighbor in $A$.

If $\mathrm{G}$ contained $\operatorname{Star}_{123}$, then $\operatorname{Star}_{123} \subseteq G(R)$ or $\quad V\left(\operatorname{Star}_{123}\right) \cap A \neq \Phi \quad$ and $V\left(\operatorname{Star}_{123}\right) \cap N \neq \Phi \quad$ and $V\left(\operatorname{Star}_{123}\right) \cap R \neq \Phi$. Because $N, R \neq \Phi$ and $\quad N \sim R, \quad\left|V\left(\operatorname{Star}_{123}\right) \cap R\right|=1 \quad$ and $\left|V\left(\operatorname{Star}_{123}\right) \cap N\right| \leq 3$.

Let $\operatorname{Star}_{123}$ be the graph with $V\left(\operatorname{Star}_{123}\right)=\{a, b, c, d . e, f, g\} \quad$ and $E\left(\operatorname{Star}_{123}\right)=\{a b, b c, c d, c e, e f, f g\} \quad$.If $a \in R$, then G-R contains the graph in Figure 2(4). If $b \in R$, then $a \in N$ and a has no neighbor in $\mathrm{A}$, which is impossible because $N=N_{G}(A)$. If $c \in R$, then $d \in N$ and $\mathrm{d}$ has no neighbor in $\mathrm{A}$, which is impossible because $N=N_{G}(A)$. If $d \in R$, then G-R contains the graph in Figure 2(2). If $e \in R$, then G-R contains the graph in Figure 2(3). If $f \in R$, then $g \in N$ and $g$ has no neighbor in A, which is impossible because $N=N_{G}(A)$. If $g \in R$, then G-R contains the graph in Figure 2(5). If $\mathrm{G}$ contained $\mathrm{Sun}_{4} \quad$ then $\mathrm{Sun}_{4} \subseteq G(R) \quad$ or $V\left(\right.$ Sun $\left._{4}\right) \cap A \neq \Phi$ or $V\left(\right.$ Sun $\left._{4}\right) \cap N \neq \Phi$ or $V\left(\right.$ Sun $\left._{4}\right) \cap R \neq \Phi$. Because $N, R \neq \Phi$ and $\quad N \sim R, \quad \mid V\left(\right.$ Sun $\left._{4}\right) \cap R \mid=1 \quad$ and $\left|V\left(\operatorname{Sun}_{4}\right) \cap N\right|=1$. If $\left|V\left(\operatorname{Sun}_{4}\right) \cap N\right|=1$, then G-R contains the graph in Figure 2(6).

\subsubsection{Proprieties of the weak-bisplit cographs}

In [9\}) is considered the number of efficient points in criteria space of multiple objective combinatorial optimization problems. Experimental results with the shortest path problem, the Steiner tree problem on graphs and the traveling salesman problem show that the number of efficient points is much lower than a polynomial upper bound. 
It is known that [14] a graph is weak-bisplit if and only if it is $\left\{P_{7}, C_{2 \mathrm{k}+1}(k \geq 2), \operatorname{Star}_{123}\right\}$-free.

Because a $P_{4}$-free graph is $P_{7}$-free, any graph $\left\{P_{4}, C_{2 k+1}(k \geq 2)\right.$, Star $\left._{123}\right\}$-free (still called weak bisplit cograph) is weak-bisplit.

Theorem 6. Let $G=(V, E)$ be a connected and incomplete graph and $(A, N, R)$ be a weak decomposition with $G(A)$ the weak component. $G$ is weak-bisplit cograph if and only if:

i) $A \sim N \sim R$

ii) $G(A), G(N), G(R)$ are weak-bisplit cographs.

Proof. Let $G$ be a weak-bisplit cograph and $(\mathrm{A}, \mathrm{N}, \mathrm{R})$ be a weak decomposition with $\mathrm{G}(\mathrm{A})$ the weak component. Then $N \sim R$, because $\mathrm{G}(\mathrm{A})$ is the weak component. Because $\mathrm{G}$ is $P_{4}$-free, $A \sim N \sim R$.

Vice versa, we assume that $\mathrm{G}(\mathrm{A}), \mathrm{G}(\mathrm{N}), \mathrm{G}(\mathrm{R})$ are weak-bisplit cographs and $A \sim N \sim R$. From [11], because $\mathrm{G}(\mathrm{A}), \mathrm{G}(\mathrm{N}), \mathrm{G}(\mathrm{R})$ are weakbisplit cographs and $A \sim N \sim R, \mathrm{G}$ is $P_{4}$-free.

$C_{2 k+1}(k \geq 2) \not \subset G(A \cup R)$, because $\mathrm{G}(\mathrm{A})$, $\mathrm{G}(\mathrm{R})$ are weak-bisplit cographs and $G(A \cup R) \quad$ is not connected. $C_{2 k+1}(k \geq 2) \not \subset G(A \cup N)$, because G(A), $\mathrm{G}(\mathrm{N})$ are weak-bisplit cographs and $A \sim N$.

$C_{2 k+1}(k \geq 2) \not \subset G(N \cup R)$, because $\mathrm{G}(\mathrm{N})$, $\mathrm{G}(\mathrm{R})$ are weak-bisplit cographs and $N \sim R$.

$C_{2 k+1}(k \geq 2) \not \subset G(A \cup N \cup R) \quad$ with $V\left(C_{2 k+1}\right) \cap A \neq \Phi, \quad V\left(C_{2 k+1}\right) \cap N \neq \Phi$, $V\left(C_{2 k+1}\right) \cap R \neq \Phi$, because $\mathrm{G}(\mathrm{A}), \mathrm{G}(\mathrm{N})$, $\mathrm{G}(\mathrm{R})$ are weak-bisplit cographs and $A \sim N \sim R$.

$\operatorname{Star}_{123} \not \subset G(A \cup R)$, because $\mathrm{G}(\mathrm{A}), \mathrm{G}(\mathrm{R})$ are weak bisplit cographs and $G(A \cup R)$ is not connected.

Star $_{123} \not \subset G(A \cup N)$, because $\mathrm{G}(\mathrm{A}), \mathrm{G}(\mathrm{N})$ are weak bisplit cographs and $A \sim N$. Star $_{123} \not \subset G(N \cup R)$, because $\mathrm{G}(\mathrm{N}), \mathrm{G}(\mathrm{R})$ are weak-bisplit cographs and $N \sim R$. Star $_{123} \not \subset G(A \cup N \cup R) \quad$ with $V\left(\operatorname{Star}_{123}\right) \cap A \neq \Phi, \quad V\left(\right.$ Star $\left._{123}\right) \cap N \neq \Phi$,
$V\left(\operatorname{Star}_{123}\right) \cap R \neq \Phi$, because $\mathrm{G}(\mathrm{A}), \mathrm{G}(\mathrm{N})$, $\mathrm{G}(\mathrm{R})$ are weak-bisplit cographs and $A \sim N \sim R$. So $\mathrm{G}$ is weak-bisplit cograph.

Next, we present how to determine the stability number, the clique number and the domination number for the weak-bisplit cographs.

Theorem 7. Let $G=(V, E)$ be a weak-bisplit cograph and $(A, N, R)$ be a weak decomposition with $G(A)$ the weak component. If $G$ is a weakbisplit cograph, then:

i) $\alpha(G)=\max \{\alpha(G(N)), \alpha(G(A))+\alpha(G(R))\}$;

ii) $\omega(G)=\omega(G(N))+\max \{\omega(G(A)), \omega(G(R))\}$;

iii) $v(G)=|N|$.

Proof.

From [12] it follows

$\alpha(G)=\max \{\alpha(G(A \cup N))$, $\alpha(G(A))+\alpha(G(R))\}$.

Because $A \sim N$, $\alpha(G(A \cup N))=\max \{\alpha(G(A)), \alpha(G(N))\}$.

So $\alpha(G)=\max \{(G(N)), \alpha(G(A))+\alpha(G(R))\}$.

Because $A \sim N \sim R$, $=\omega(G(N))+\max \{\omega(G(A)), \omega(G(R))\}$.

Because $A \sim N \sim R, \mathrm{~N}$ is cutest minimal, so a domination set of minimal cardinal is $\mathrm{N}$ that is $v(G)=|N|$.

\subsection{Algorithm for the determination of $\omega(G)$ in a weak-bisplit cograph $G$}

Input: A weak-bisplit cograph, $G=(V, E)$.

Output: The determination of $\omega(G)$.

1. The determination of a weak decomposition $(A, N, R)$ with $G(A)$ the weak component.

2. We denote by $Q_{A} \subseteq A, Q_{N} \subseteq N, Q_{R} \subseteq R, \quad \mathrm{a}$ clique of maximum cardinal from $A, N, R$.

3. The determination of the degree of each vertex in $G$.

4. The maximum degree in $G$ is in $Q_{A}$ or $Q_{N}$ or $Q_{R}$. 
5. Let $v$ be a vertex of maximum degree in $G$.

If $v$ is in $N$, then 6 follows and then 9 , so that, if $\mathrm{v}$ is in $A$, then 7 follows and then 9 , otherwise 8 follows and then 9 .

6. Then $Q_{N}$ is the set consisting of all the vertices in $N$ with the same degree as $v$ and $\omega(G(N))=\left|Q_{N}\right|$.

Let $H=G-N=G(A \cup R)$.

The degrees of the vertices in $H$ verify:

$d_{H}(a)=d_{G}(a)-|N|, \forall a \in A$;

$d_{H}(r)=d_{G}(r)-|N|, \forall r \in R$.

Let $w$ be the vertex of maximum degree in $H$.

If $w$ is from $A$ then 6.1 otherwise 6.2.

6.1. $Q_{A}$ consists of the set of vertices in $A$ of the same degree as $w$ and $\omega(G(A))=\left|Q_{A}\right|$.

Let $F=H-A=G(R)$.

The degrees of the vertices in $F$ verify:

$d_{F}(r)=d_{H}(r)=d_{G}(r)-|N|, \forall r \in R$.

Let $u$ be the vertex of maximum degree in $F$.

We have $Q_{R}$ consisting of the set of vertices in $R$ of the same degree as $u$ and $\omega(G(R))=\left|Q_{R}\right|$.

6.2. $Q_{R}$ consists of the set of vertices in $R$ of the same degree as $w$ and $\omega(G(R))=\left|Q_{R}\right|$.

Let $F=H-R=G(A)$.

The degrees of the vertices in $F$ verify:

$d_{F}(a)=d_{H}(a)=d_{G}(a)-|N|, \forall a \in N$.

Let $u$ be the vertex of maximum degree in $F$.
We have $Q_{A}$ consisting of the set of vertices in $A$ of the same degree as $u$ and $\omega(G(A))=\left|Q_{A}\right|$.

7. Then $Q_{A}$ is the set consisting of all the vertices in $A$ of the same degree $v$ and $\omega(G(A))=\left|Q_{A}\right|$.

Let $H=G-A=G(N \cup R)$.

The degrees of the vertices in $H$ verify:

$d_{H}(n)=d_{G}(n)-|A|, \forall n \in N$;

$d_{H}(r)=d_{G}(r), \forall r \in R$.

Let $w$ be the vertex of maximum degree in $H$.

If $w$ is from $N$, then 7.1. otherwise 7.2.

7.1. $Q_{N}$ consists of the set of vertices in $N$ of the same degree as $w$ and $\omega(G(N))=\left|Q_{N}\right|$.

Let $F=H-N=G(R)$.

The degrees of the vertices in $F$ verify:

$d_{F}(r)=d_{H}(r)-|\Lambda|=d_{G}(r)-|N|, \forall r \in R$.

Let $u$ be the vertex of maximum degree in $F$.

We have $Q_{R}$ containing of the set of vertices in $R$ of the same degree as $u$ and $\omega(G(R))=\left|Q_{R}\right|$.

7.2. $Q_{R}$ contains of the set of vertices in $R$ of the same degree as $w$ and $\omega(G(R))=\left|Q_{R}\right|$.

Let $F=H-R=G(N)$.

The degrees of the vertices in $F$ verify: $\begin{aligned} d_{F}(n) & =d_{H}(n)-|R|= \\ & =d_{G}(n)-(|A|+|R|), \forall n \in N\end{aligned}$.

Let $u$ be the vertex of maximum degree in $F$.

We have $Q_{N}$ containing the set of vertices in $N$ of the same degree as $u$ and $\omega(G(N))=\left|Q_{N}\right|$. 
8. Then $Q_{R}$ is the set containing all the vertices in $R$ of the same degree as $v$ and $\omega(G(R))=\left|Q_{R}\right|$.

Let $H=G-R=G(A \cup N)$.

The degrees of the vertices in $H$ verify:

$d_{H}(a)=d_{G}(a), \forall a \in A$;

$d_{H}(n)=d_{G}(n)-|R|, \forall n \in N$.

Let $w$ be the vertex of maximum degree in $H$.

If $w$ is from $A$ then 8.1 otherwise 8.2 .

8.1. $Q_{A}$ consists of the set of vertices in $A$ of the same degree as $w$ and $\omega(G(A))=\left|Q_{A}\right|$.

Let $F=H-A=G(N)$.

The degrees of the vertices in $F$ verify:

$$
\begin{aligned}
d_{F}(n) & =d_{H}(n)-|A|= \\
& =d_{G}(n)-(|A|+|R|), \forall n \in N
\end{aligned} .
$$

Let $u$ be the vertex of maximum degree in $F$.

We have $Q_{N}$ consisting of the set of vertices in $N$ of the same degree as $u$ and $\omega(G(N))=\left|Q_{N}\right|$.

8.2. $Q_{N}$ consists of the set of vertices in $N$ of the same degree as $w$ and $\omega(G(N))=\left|Q_{N}\right|$.

Let $F=H-N=G(A)$.

The degrees of the vertices in $F$ verify:

$$
\begin{aligned}
d_{F}(a) & =d_{H}(a)-|N|= \\
& =d_{G}(a)-|N|, \forall a \in A
\end{aligned} .
$$

Let $u$ be the vertex of maximum degree in $F$.

We have $Q_{A}$ consisting of the set of vertices in $A$ of the same degree as $u$ and $\omega(G(A))=\left|Q_{A}\right|$.

9. $\omega(G)=\omega(G(N))+$

$$
+\max \{\omega(G(A)), \omega(G(R))\}
$$

Remark 1. Because the block that requires the longest execution time, $O(n+m)$, is the determination of the weak decomposition $(A, N, R)$, we conclude that total execution time is $O(n+m)$.

\section{Conclusions and Future Work}

In this paper, using weak decomposition, we characterize the weak-bisplit graphs, as well as some of their subclasses. Also, we give an $O(n+m)$ of how to determine the density of a weak-bisplit graph and we directly calculate the domination number for this class of graphs.

In the future papers we will establish the proprieties of geometric graphs.

\section{REFERENCES}

1. BRANDSTAEDT, A., P. L. HAMMER, V. B. LE, V. V. LOZIN, Bisplit Graphs, Rutcor Research Report RRR 28-2002, http://rutcor.rutgers.edu/pub/rrr/reports20 02/28_2002.ps.

2. BERGE, C., Graphs, North-Holland, Amsterdam. 1985.

3. FOUQUET, J. L., V. GIAKOUMAKIS, J. M. VANHERPE, Bipartite Graphs Totally Decomposable by Canonical Decomposition, Intl. J. Foundations Comput. Sci. 10, 1999, pp. 513-533.

4. FOUQUET, J. L., J. M. VANHERPE, On Bipartite Graphs with Weak Density of Some Subgraphs, Discrete Mathematics 307, 2007, pp. 1516-1524.

5. GIAKOUMAKIS, V., J. M. VANHERPE, Linear Time Recognition of Weak Bisplit Graphs, Electronic Notes in Discrete Mathematics, 5, 2000, pp. 138-141.

6. GOLUMBIC, M. C., R. UDI, On the Clique-width of Perfect Graph Classes (extended abstract), Lect. Notes Comput. Sci. 1665, 1999, pp. 135-147.

7. HAIKO, M, A. BRANDSTAEDT, The NP-completeness of Steiner Tree and Dominating Set for Chordal Bipartite Graphs, Theor. Comput. Sci. 53, 1987, pp. 257-265. 
8. POLJAK, S., A Note on the Stable Sets and Coloring of Graphs, Comment. Math. Univ. Carolin. 15, 1974, pp. 307-309.

9. STANOJEVIC, M., M. VUJOŠEVIC, B. STANOJEVIC, Computation Results of Finding All Efficient Points in Multiobjective Combinatorial Optimization, Intl. J. of Computers, Communications \& Control, ISSN 18419836, E-ISSN 1841-9844, Vol. III, 2008, No. 4, pp. 374-383.

10. TALMACIU, M., Decomposition Problems in the Graph Theory with Applications in Combinatorial Optimization - Ph. D. Thesis, University "Al. I. Cuza" Iasi, Romania, 2002.
11. TALMACIU, M., E. NECHITA, Recognition Algorithm for Diamondfree Graphs, Informatica, 18, 2007, pp. 457-462.

12. TALMACIU, M; E. NECHITA, G. C. CRISAN, A Recognition Algorithm for a Class of Partitionable Graphs that Satisfies the Normal Graph Conjecture, Studies in Informatics and Control, 18, 2009, pp. 349-354.

13. http://wwwteo.informatik.unirostock.de/isgci/classes/gc_444.html

14. http://wwwteo.informatik.unirostock.de/isgci/classes/gc_457.html 